\title{
Molecular Characterization of Peritoneal Involvement in Primary Colon and Ovary Neoplasm: The Possible Clinical Meaning of the P2X7 Receptor-Inflammasome Complex
}

\author{
Anna Solini $^{a}$ Luigi Cobuccio $^{a}$ Chiara Rossi $^{\mathrm{a}}$ Federico Parolini $^{\mathrm{b}}$ \\ Edoardo Biancalana $^{b}$ Stefania Cosio $^{b}$ Massimo Chiarugi ${ }^{a}$ Angiolo Gadducci $^{b}$ \\ ${ }^{a}$ Department of Surgical, Medical, Molecular and Critical Area Pathology, University of Pisa, Pisa, Italy; ${ }^{b}$ Department \\ of Clinical and Experimental Medicine, University of Pisa, Pisa, Italy
}

\section{Keywords}

Colon cancer - Ovarian cancer · Peritoneal carcinomatosis . P2X7 receptor $\cdot$ NLRP3 inflammasome

\begin{abstract}
Introduction: Colon cancer (CC) and epithelial ovarian cancer (EOC) are common and severe neoplasms frequently sharing a massive inflammatory involvement of peritoneum. A detailed molecular characterization of such carcinomatosis has not been performed, so far. Methods: Omental adipocytes were isolated from thirty-three adult women who underwent primary surgery for CC or EOC. Expression of several pro-inflammatory genes was determined by real-time PCR and immunofluorescence. Data were related to the clinical phenotype of the patients. Results: CD68, FGFR1, and IL-6 were significantly more expressed in adipocytes from CC patients and VEGF in adipocytes from EOC. TNFa, TGF $\beta$, or MCP-1, as well as the pro-inflammatory platform P2X7R-NL$\mathrm{RP} 3$, did not differ between the 2 cancers. White blood cell count, mirroring systemic inflammation, was related to adi-
\end{abstract}

pocyte P2X7R $(R=0.508, p=0.003)$, NLRP3 $(R=0.405 ; p=$ $0.02)$, and MCP-1 $(R=0.448 ; p=0.009)$. P2X7R and NLRP3 were the only inflammatory factors significantly more expressed in patients carrying both omental and peritoneal carcinosis, who were also characterized by a higher leukocytosis. None of the tested inflammatory markers was associated with tumor grading for both neoplasms; however, the presence of metastases was associated with a higher adipocyte expression of FGFR1 and TGF $\beta$. Conclusion: We show here that rarely measured molecules seem to specifically characterize omental carcinomatosis of CC or EOC, while more common inflammatory agents like TNFa, TGF $\beta$, or MCP-1 do not; the P2X7R-NLRP3 complex marks omental and peritoneal carcinosis and is related to circulating white blood cells and MCP-1, involved in monocyte-macrophage tissue infiltration; increased TGF $\beta$ and FGFR1 characterize the tumoral dissemination.

(c) 2021 S. Karger AG, Basel

Massimo Chiarugi and Angiolo Gadducci are sharing last authorship.
Correspondence to:

Anna Solini, anna.solini@med.unipi.it 


\section{Introduction}

The GLOBOCAN 2018 database, which estimated the worldwide incidence and mortality for 36 cancers in 185 countries, reported 295,414 new cases of epithelial ovarian cancer (EOC) and 1,096,601 new cases of colon cancer (CC) and, respectively, 184,799 and 551,269 deaths due to these malignancies in 2018 [1]. These 2 neoplasms share a relevant peritoneal involvement. The term "peritoneal carcinomatosis" (PC), usually indicating the shedding, implantation, and dissemination of a tumor to the peritoneal coating that covers the abdominal cavity and the viscera within it, is the result of a complex sequence of molecular events by which tumor cells disseminate from their primary organ to establish independent metastatic deposits on the visceral and parietal lining of the abdominal cavity [2]. The presence of this condition invariably indicates an advanced stage of disease, associated with a very poor prognosis; moreover, peritoneal metastases are often associated with the formation of malignant ascites, resulting in higher morbidity due to related intraabdominal hypertension conditions. The incidence of PC is unknown, due to low sensitivity of preoperative imaging techniques and heterogeneity of published findings and biological behavior of the primary tumor [3]; in fact, the gold standard in diagnosing PC continues to be the direct peritoneal surgical visualization. The understanding of the molecular events involved in peritoneal metastatic spread, and the possibility of interrupting their devastating sequence, is therefore of paramount importance to find new therapeutic strategies. To date, albeit still incomplete, main evidence comes from the understanding of the biology and pathways of peritoneal dissemination of these 2 frequent intra-abdominal tumors.

Transcoelomic spread is the most traveled road that gives origin to synchronous peritoneal metastases in patients with locally advanced intraperitoneal CC as a result of full-thickness invasion of the bowel wall by an invasive cancer [4]. The process of transcoelomic seeding is the consequence of a molecular cross talk between cancer cells and host elements, involving several steps, known as the "peritoneal metastatic cascade" [5]. Several studies in the last decade have shown that inflammatory mediators promote not only carcinogenesis but also tumor progression. The systemic inflammatory response, usually made of composite ratios or cumulative scores of different circulating white blood cells (WBCs) or acute phase proteins, has shown some prognostic significance [6]; the production of these mediators participates in the upregulation of adhesion molecules on the peritoneal surface [7-9], thus en- hancing the metastatic potential of tumor cells. In this view, an intriguing topic concerns the relationship between carcinosis and the ATP-sensitive P2X7 receptor (P2X7R), a powerful and multifaceted inflammatory mediator $[10,11]$. In most tumors, P2X7R is constantly and tonically active, thus stimulating cell growth, proliferation, and migration, and influencing tumor microenvironment $[12,13]$; however, this receptor can also act as a nonredundant host factor in in vivo anticancer response [14].

For EOC, the formation of intraperitoneal metastases is driving increasing attention: such process requires a permissive microenvironment within the peritoneal cavity, and ascites components are mainly responsible for such scenario, suppressing peritoneal inflammatory reactions inducing angiogenesis and promoting cancer cell proliferation and migration $[15,16]$. P2X7R has been poorly investigated in the human ovary; it is expressed in the surface epithelium and maintained in EOC [17], but its putative role as biomarker or even as disease mediator has never been explored. Indeed, the relevance of peritoneal surface infiltration as compared with organ invasion is still debated, and a detailed and comparative characterization of the inflammatory profile of peritoneum in these 2 tumors has not been performed, neither related to the prognosis of these patients; the present study was aimed at addressing this specific issue.

\section{Subjects and Methods}

\section{Study Design and Ethical Approval}

This prospective study was approved by the institutional review board of the University of Pisa (\#13831). Participants signed a written informed consent for the provision of biological material for research purposes.

\section{Patients}

Fifty-two female adult patients aging 40-80 years, with clinical indication for primary surgical treatment for intraperitoneal CC or EOC in the section of General Surgery and Gynecologic Oncology of the University Hospital in Pisa, Italy, between January 2016 and June 2018, were consecutively enrolled on a volunteer basis. Clinical history and serum/plasma samples for biochemistry and measurement of inflammatory markers were collected 2 days before the surgery. Pathological tumor staging was performed according to the American Joint Committee on Cancer staging manual [18] and according to the FIGO staging system for EOC [19]. Biochemical variables were measured by standard methods on fasting blood samples collected $48 \mathrm{~h}$ before surgery.

To exclude tumors with invasion limited to only the most superficial layers of the colon and ovary, inclusion criteria for patients with intraperitoneal CC were the histologically confirmed diagnosis of adenocarcinoma with a tumor invasion through at least muscularis propria and into subserosa (stage T3 according to 
TNM staging system), regardless of the presence of peritoneal or metastatic diffusion of the disease. Regarding patients undergoing surgery for EOC, inclusion criteria were a postoperative histologically confirmed diagnosis of EOC with involvement at least of ovarian serosa (stage Ic according to FIGO staging system), regardless of the presence of peritoneal carcinosis. Exclusion criteria were the presence of intra-abdominal inflammatory disease, previous neoadjuvant chemotherapy or radiotherapy, concurrent steroidal anti-inflammatory therapy, and immunosuppressive states.

The final population consisted of 15 patients with histological diagnosis of EOC and 18 patients with intraperitoneal CC. Causes of exclusion were the mismatch between the histological inclusion criteria and the tumoral staging (parameter T) on anatomic-pathological examination ( 9 patients), and the scarce amount of RNA extracted from tissue samples, insufficient to perform a molecular analysis (10 patients). We also enrolled a control group of 5 female patients undergoing surgical treatment for intraperitoneal benign disease without surgical evidence of intra-abdominal inflammatory or infection condition, to normalize data of gene expression obtained in the study cohort.

\section{Tissue Specimens}

Immediately after starting the surgical procedure, 2 samples of disease-free omentum were collected in each patient, stored in $1 \mathrm{X}$ Hank's balanced salt solution (H4385, Sigma-Aldrich, Merck Life Science, Milan, Italy), and sent to the laboratory for molecular processing: 1 sample was used for adipocyte isolation and the other for immunofluorescence experiment. For the mature adipocyte isolation, 1 tissue sample was digested with collagenase type II (C6885, Sigma-Aldrich) at $37^{\circ} \mathrm{C}$ in Hank's solution for $1 \mathrm{~h}$. After centrifugation at $700 \mathrm{~g}$, the superficial oil was removed, the suspension was filtered (100 $\mu \mathrm{m}$ filter), and cells were repeatedly washed with phosphate buffer solution (PBS) before storing them at $-80^{\circ} \mathrm{C}$. The specimen for immunofluorescence was fixed in $10 \%$ neutral buffered formalin (F0043, Diapath, Martinengo, Italy) for $2 \mathrm{~h}$ at room temperature, washed in PBS, and cryoprotected in 30\% sucrose overnight at $4^{\circ} \mathrm{C}$. The next day sample was included in H-OCT compound (R0030, Histo-Line Laboratories, Milan, Italy) and stored at $-20^{\circ} \mathrm{C}$ until sectioning in cryostat instrument.

\section{RNA Extraction and Retrotranscription}

RNA extraction from isolated adipocytes was performed in approximately $500 \mu \mathrm{L}$ of adipocytes lysed in $350 \mu \mathrm{L}$ of RLT buffer with $\beta$-mercaptoethanol using the TissueLyser (Qiagen, Hilden, Germany): in this instrument, disruption and homogenization are achieved through the beating and grinding effect of steel beads on the sample, during a shacking step at $30 \mathrm{~Hz}$ for $2 \mathrm{~min}$. After a centrifugation step to remove insoluble material, the supernatant was carefully transferred in new tubes and loaded in the QIAcube instrument following the manufacturing protocol. QIAcube (Qiagen) is a robotic workstation for automated purification of nucleic acids, equipped with RNeasy mini kit (Qiagen). After a spectrophotometric quantification (by NanoDrop 2000c, Thermo Fisher Scientific, Waltham, MA, USA), 750 ng of total RNA was reverse transcribed to single-stranded cDNA by high-capacity cDNA reverse transcription kit (Applied Biosystems, Thermo Fisher Scientific, Waltham, MA, USA).

\section{Quantitative Real-Time PCR}

To compare the tissue inflammatory profile of the 2 diseases, we measured adipocyte gene expression of TNFa (key mediator of
Table 1. Clinical characteristics of the study participants

\begin{tabular}{lll}
\hline & CC $(n=18)$ & EOC $(n=15)$ \\
\hline Age, years & $73.6 \pm 8.8$ & $72.0 \pm 8.5$ \\
$\mathrm{BMl}, \mathrm{kg} / \mathrm{m}^{2}$ & $23.8 \pm 3.5$ & $25.4 \pm 4.4$ \\
$\mathrm{WBCs}, \mathrm{n} / \mathrm{mmc}$ & $7.64 \pm 2.91$ & $8.22 \pm 2.02$ \\
Red blood cells, n/mmc & $4.5 \pm 1.8$ & $4.5 \pm 0.6$ \\
$\mathrm{Hb}, \mathrm{g} / \mathrm{dL}$ & $10.9 \pm 1.7$ & $12.9 \pm 1.8$ \\
Platelets, $10^{3} / \mathrm{mmc}$ & $310 \pm 77$ & $293 \pm 87$ \\
Fasting glucose, mg/dL & $102 \pm 28$ & $103 \pm 20$ \\
Ca125, kU/mL & $15 \pm 11$ & $1,210 \pm 1,820$ \\
Omental carcinosis, \% & 11.1 & 53.3 \\
Peritoneal carcinosis, \% & 5.5 & 66.6 \\
Stage 3-4, \% & 55.6 & 66.7 \\
Metastases, \% & 11.1 & 6.7 \\
\hline
\end{tabular}

WBCs, white blood cells; CC, colon cancer; EOC, epithelial ovarian cancer.

inflammatory response and apoptosis in cancer), TGF $\beta 1$ (involved in ovarian cell migration), IL-6 and IL-2 (pro-inflammatory adipocytokines), VEGF (a major determinant of vascular permeability and angiogenesis), CCL2/MCP-1 (playing a role in neoplastic progression), CD68 (related to cell proliferation), CD73 (upregulated in CC and EOC, and associated with tumor neovascularization, angiogenesis, and chemoresistance), and FGFR1 (hyper-expressed in several malignancies). We also explored the P2X7R and the NLRP3 inflammasome complex, a platform involved in the modulation of chemotactic factors and acute phase cytokines.

Gene expression analysis was executed in real-time PCR using the TaqMan Assays (Thermo Fisher Scientific): they consist of a target-specific probe flanked by a pair of PCR primers, and the probe is conjugated with a fluorescence dye on the $5^{\prime}$-end and a quencher on the $3^{\prime}$-end. Triplicate reactions were prepared for each sample according to the TaqMan standard protocol. Quantification of gene expression was performed using the $\Delta \Delta \mathrm{C}_{t}$ calculation, where $\mathrm{C}_{\mathrm{t}}$ is the threshold cycle. The amount of the target gene, normalized by housekeeping gene ACTB and relative to the calibrator, is given as $2^{-\Delta \Delta \mathrm{Ct}}$ : the calibrator was a pool of adipocytes isolated from a group of control patients surgically managed for intraperitoneal benign disease without evidence of peritoneal inflammation. Online supplementary Table A (for all online suppl. material, see www.karger.com/doi/10.1159/000519690) reports TaqMan assay codes.

\section{Immunofluorescence}

Cryostat sections of omental tissue after 3 wash with PBS were reacted $1-2 \mathrm{~h}$ at room temperature with a blocking solution (5\% BSA and $0.3 \%$ Triton X-100 in PBS) to saturate aspecific binding sites; samples were then incubated overnight at $4^{\circ} \mathrm{C}$ with specific primary antibodies (P2X7R: APR-004, Alomone Labs, Jerusalem, Israel, 1:100 and NRLP3: AG-20B-0014, Adipogen AG, Liestal, Switzerland, 1:200) diluted in PBS containing 1\% BSA and $0.1 \%$ Triton X-100. Immunoreactivity was revealed with secondary antibodies against mouse or rabbit antibody conjugated with fluorescence molecules (Alexa Fluor 594-goat@rabbit and 488@mouse antibodies, Invitrogen, Thermo Fisher Scientific, Waltham, MA,
Solini/Cobuccio/Rossi/Parolini/ Biancalana/Cosio/Chiarugi/Gadducci 
Fig. 1. Expression of genes differentially expressed in adipocytes isolated from omental samples of CC and EOC patients. All determinations have been performed in duplicate. Data were compared by Mann-Whitney U test. CC, colon cancer; EOC, epithelial ovarian cancer.
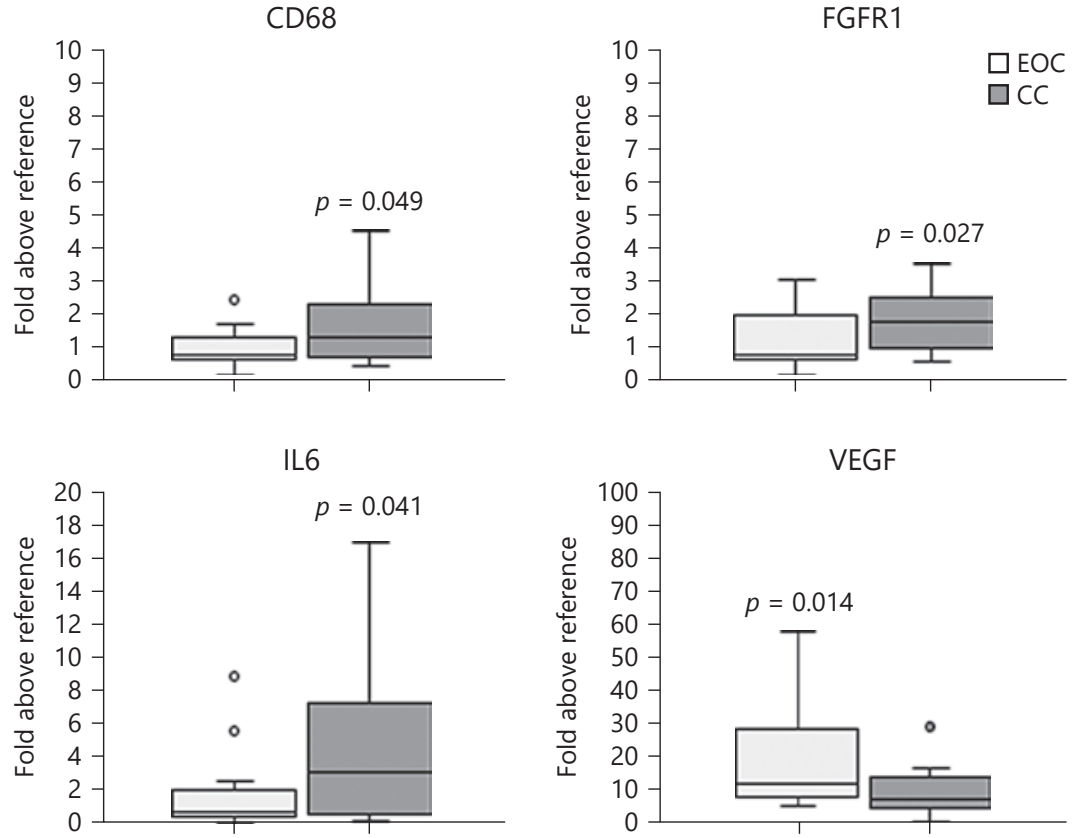

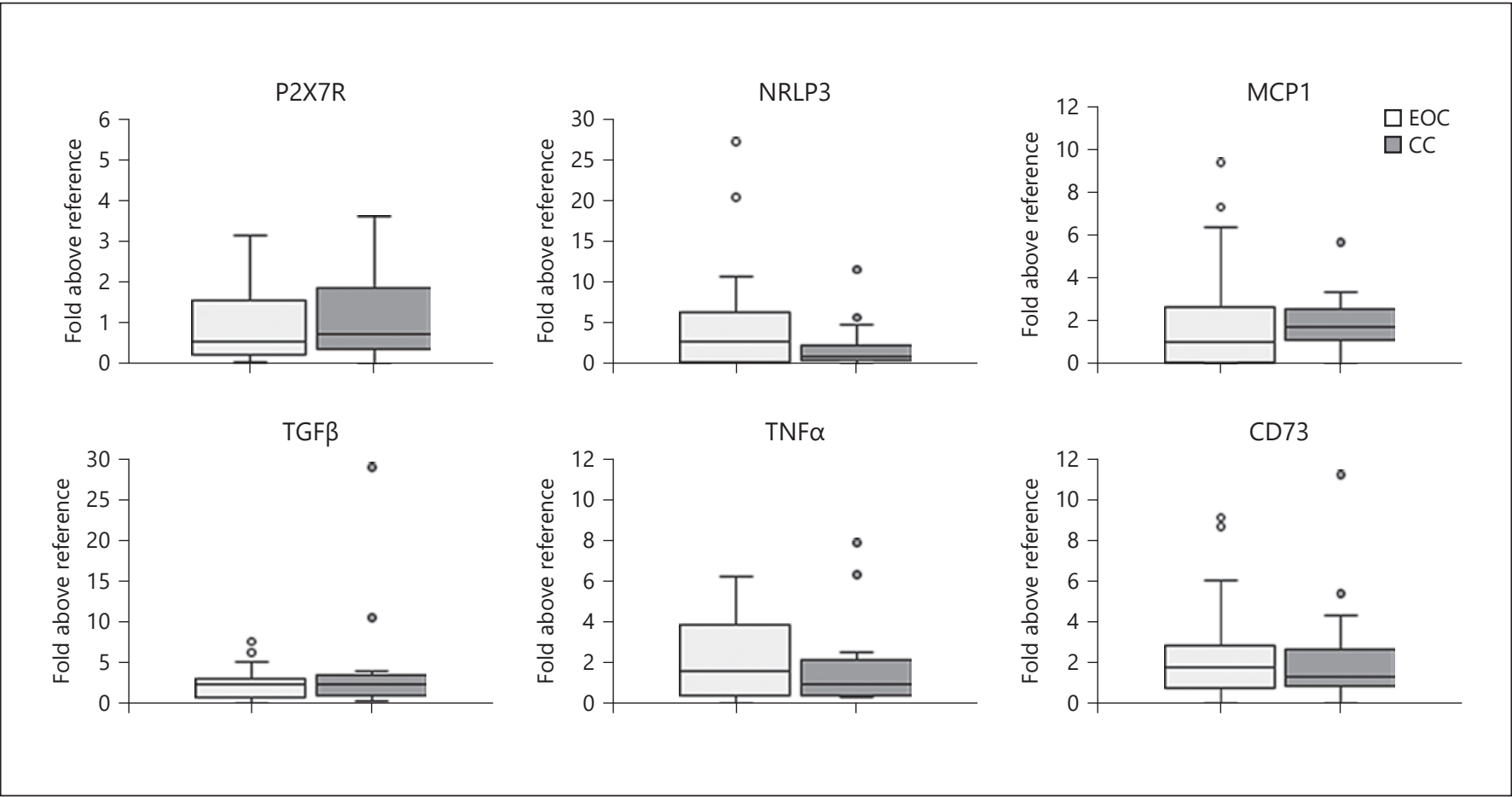

Fig. 2. Expression of genes not differentially expressed in adipocytes isolated from omental samples of CC and EOC patients. All determinations have been performed in duplicate. Data were compared by Mann-Whitney U test. CC, colon cancer; EOC, epithelial ovarian cancer; P2X7R, P2X7 receptor. 


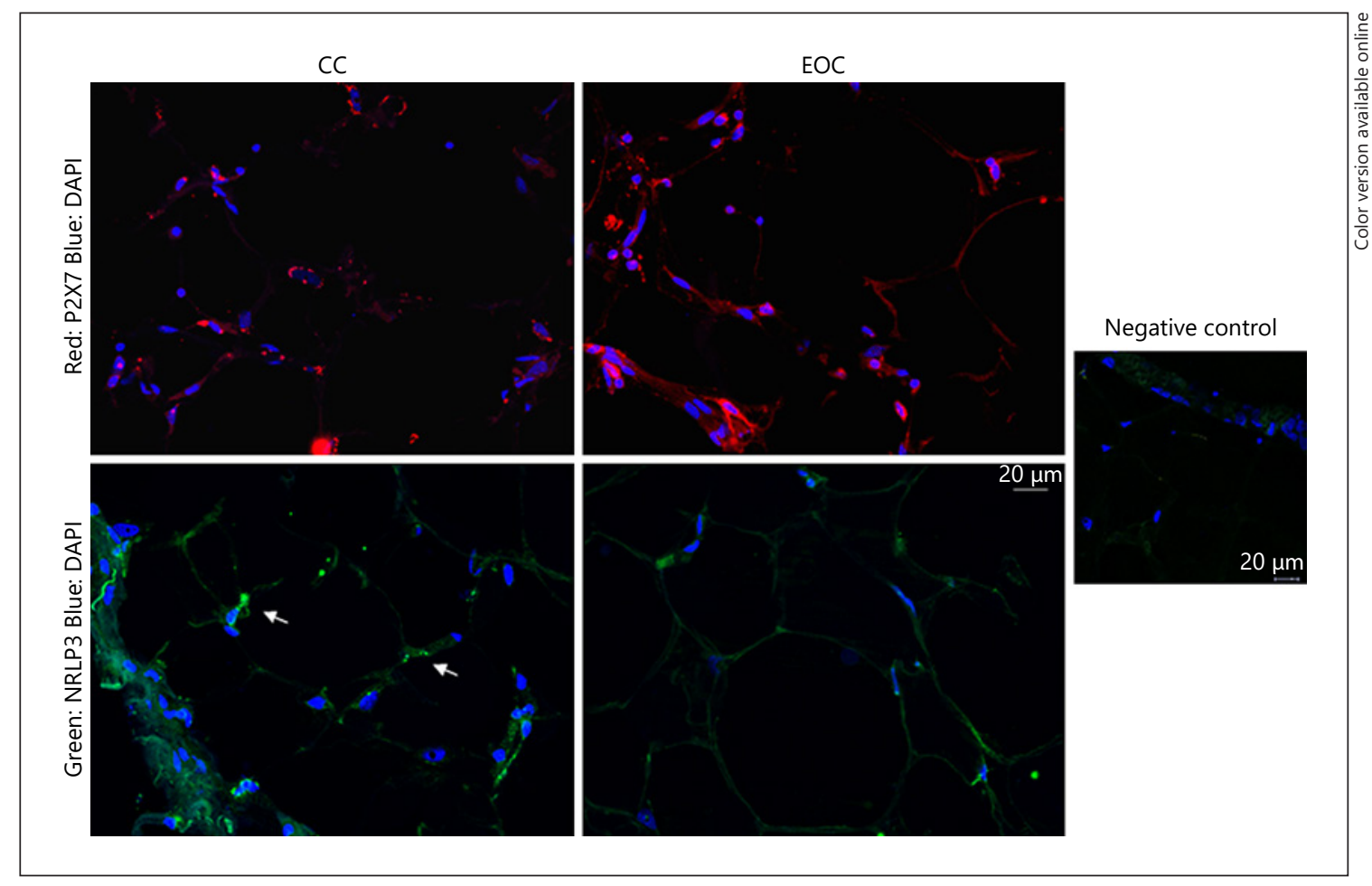

Fig. 3. Representative image of P2X7R and NRLP3 immunoreactivity in adipocytes from omental tissue sections of CC and EOC patients. In controls, only secondary antibodies were used. P2X7R, P2X7 receptor; CC, colon cancer; EOC, epithelial ovarian cancer.

USA). The images were acquired with a Leica TCS SP8 confocal microscope (Leica, Wetzlar, Germany). After a preliminary analysis of different samples to establish the best conditions to obtain an ideal instrumental performance, the confocal setting was held constant within all experimental sessions.

\section{Statistical Analysis}

Results are expressed as mean $\pm \mathrm{SD}$, unless indicated. MannWhitney $U$ test was used to perform a comparison of not normally distributed data. Pearson's $\chi^{2}$ or Fisher's exact tests were used to compare categorical qualitative variables, where appropriate. Relationships between parameters were tested by Spearman correlation coefficient. A $p$ value $<0.05$ was considered statistically significant.

\section{Results}

Clinical characteristics of the 2 study groups are shown in Table 1. The 2 subsets of patients were comparable for age, BMI, and WBC count; hemoglobin was lower in CC. Peritoneal and omental carcinosis was present in 10 and 8 patients with EOC and 1 and 2 patients with intraperitoneal $\mathrm{CC}$, respectively.

\section{Expression of the Inflammatory Markers in Omental} Adipocytes

Figures 1 and 2 show gene expression of the inflammatory markers in omental adipocytes. A partially differential expression pattern was observed, with a significantly higher expression of CD68, FGFR1, and IL-6 in adipocytes from CC patients and enhanced VEGF expression in adipocytes from EOC (Fig. 1). The expression of other generic markers of tissue inflammation, matrix deposition, and cell migration, like TNF $\alpha$, TGF $\beta$, or MCP-1, did not differ between the 2 cancers (Fig. 2). Similarly, the inflammatory platform P2X7R-NLRP3 did not differ, with P2X7R barely expressed in both and NLRP3 showing an ample variability. We then compared the expression of some of these biomarkers between controls and EOC patients, dividing the latter according to the presence or absence of PC and omental carcinomatosis. Data are shown in online supplementary Fig. A: although characterized by a large variability, both groups showed a significantly higher expression of VEGF versus controls $(p<0.05)$.

Figure 3 confirmed the expected localization of P2X7R and NLRP 3 on the plasma membrane of adipocytes from 
Table 2. Matrix of univariate correlations between clinical parameters and inflammatory biomarkers (statistically significant in bold)

\begin{tabular}{|c|c|c|c|c|c|c|c|c|c|c|c|c|}
\hline WBC & - & & & & & & & & & & & \\
\hline $\mathrm{Hb}$ & 0.078 & - & & & & & & & & & & \\
\hline P2X7R & 0.508 & -0.255 & - & & & & & & & & & \\
\hline NLRP3 & 0.405 & 0.209 & 0.001 & - & & & & & & & & \\
\hline MCP1 & 0.448 & 0.161 & 0.181 & 0.658 & - & & & & & & & \\
\hline VEGF & -0.302 & 0.472 & 0.116 & 0.036 & 0.078 & 0.252 & -0.062 & - & & & & \\
\hline FGFR1 & 0.029 & 0.011 & 0.054 & -0.216 & -0.222 & 0.318 & 0.592 & 0.056 & - & & & \\
\hline IL-6 & 0.143 & -0.252 & -0.111 & 0.158 & 0.187 & -0.189 & -0.017 & -0.169 & -0.133 & - & & \\
\hline CD68 & 0.072 & 0.215 & 0.359 & -0.001 & 0.055 & 0.130 & 0.130 & -0.116 & 0.506 & -0.111 & - & \\
\hline CD73 & -0.089 & 0.308 & 0.098 & 0.310 & 0.050 & 0.540 & 0.314 & 0.324 & 0.150 & -0.019 & & - \\
\hline
\end{tabular}

WBCs, white blood cells; P2X7R, P2X7 receptor.

CC and EOC, with no significant difference between the 2 tumors. The plasma membrane localization of NLRP3 in adipocyte seems to be cell-specific, being intracytoplasmic in inflammatory circulating cells (data not shown).

\section{Relation of the Inflammatory Markers with WBCs}

Table 2 shows linear correlations between WBC and different inflammatory molecules in the whole study group. In terms of pathophysiologic mechanisms, the most interesting ones appear those linking MCP-1 with NLRP3 $(R 0.658, p=0.001)$ and those linking FGFR1 with CD68 $(R 0.506, p=0.0027)$ and TGF $\beta$ with TNFa $(R$ $0.543, p=0.0011)$; the former was reinforced by the observation of how WBC, a gross indicator of systemic inflammation, was related to adipocyte $\mathrm{P} 2 \mathrm{X} 7 \mathrm{R}(R=0.508$, $p=0.003)$, NLRP3 $(R=0.405, p=0.02)$, and MCP- $1(R=$ $0.448, p=0.009)$, confirming the functional link between such inflammatory platform in adipocytes and lymphomonocyte recruitment and chemotaxis [20]. Scatterplots of all the tested linear correlations are shown in online supplementary Fig. B: even when correlations appear weak or driven by a few subjects, data are quite homogeneously distributed when log-transformed.

\section{Relation of Inflammatory Markers with the Clinical \\ Phenotype}

Distribution of these markers according to omental and peritoneal carcinosis was tested. Interestingly, P2X7R and NLRP3 were the only inflammatory factors significantly more expressed in patients carrying both types of carcinosis; data are shown in Figure 4a, b. Noteworthy, WBC showed the same behavior, being significantly higher $(p<$

P2X7R-Inflammasome and Peritoneal Carcinomatosis
0.01 ) in patients who developed omental or peritoneal carcinosis (Fig. 4c). As expected, circulating Ca125, whose levels in CC and EOC are reported in Table 1, was significantly higher in EOC patients who developed peritoneal carcinosis $(192 \pm 115$ vs. $1,838 \pm 2,090 \mathrm{kU} / \mathrm{mL}, p<0.001)$.

\section{Relation of the Inflammatory Markers with the}

\section{Disease Stage}

We also checked whether these inflammatory markers would be differently expressed according to the disease stage; as shown in online supplementary Fig. C, none of them was associated with cancer grading in both neoplasms. Conversely, the presence of metastases was associated with a higher adipocyte expression of FGFR1 and TGF $\beta$ (online suppl. Fig. D).

\section{Discussion}

We provide here a comparative characterization of peritoneal and omental expression of several inflammatory molecules potentially involved in the pathogenesis of colorectal and ovary cancer. The main observations are the following: (i) some rarely measured molecules seem to specifically characterize adipocytes of a given tumor, while more common inflammatory agents like TNF $\alpha$ or TGF $\beta$ or MCP-1 do not differ between the 2 tumors; (ii) the P2X7R-NLRP3 inflammasome, even not extremely abundant and not differently expressed in the gut and in the ovary, is higher in adipocytes of patients with carcinomatosis and shows a strong relationship with circulating WBC and with the chemotactic factor MCP-1, in- 


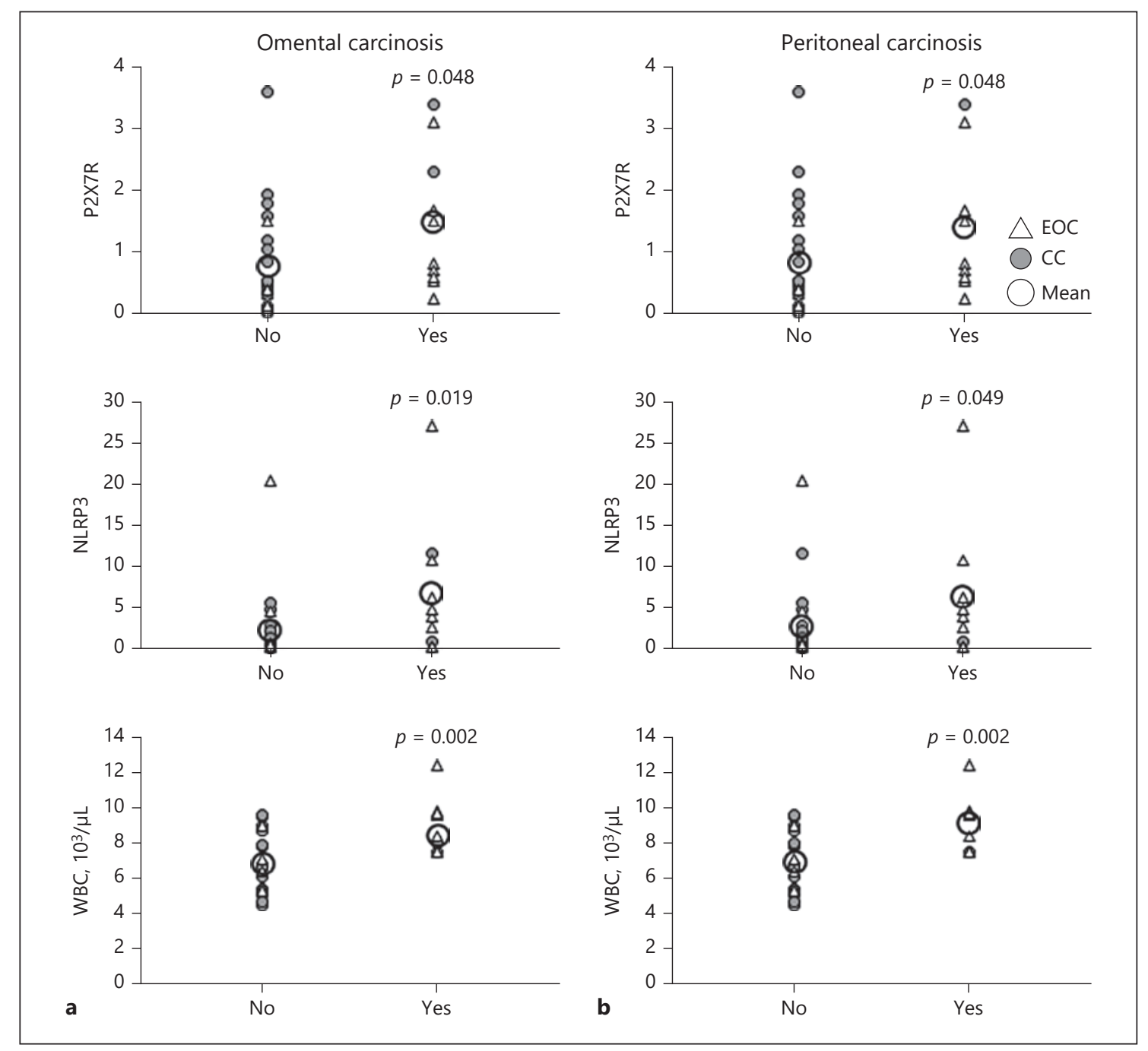

Fig. 4. $P 2 X 7 R$ and NLRP3 gene expression and peripheral WBC in patients with and without carcinosis in omental tissue (a) and peritoneum (b). All determinations have been performed in duplicate. Data were compared by Mann-Whitney U test. P2X7R, P2X7 receptor; WBC, white blood cell; CC, colon cancer; EOC, epithelial ovarian cancer.

volved in monocyte-macrophage tissue infiltration; (iii) increased omental TGF $\beta$ and FGFR1 are significantly related to tumor dissemination.

CC adipocytes display a higher expression of IL-6, CD68, and FGFR1. IL-6, abundantly represented in colon adipocytes, promotes tumor cell adhesion to a mesothelial monolayer and can directly induce vessel sprouting, as well as endothelial cell proliferation and migration, with similar potency to VEGF [21]. The increased CD68 (recognized as a macrophage marker) is intriguing, at least in light of a previous report suggesting that high infiltration of $\mathrm{CD} 8^{+}$could be a favorable prognostic marker in CC [22]. Our results are at odds with this one, and in our study group, CD68 does not correlate with prognosis or dissemination of the tumor. FGFR1 gene alterations have been reported in a wide variety of cancers, including those of the urinary bladder, breast, ovary, prostate, endometrium, lung, and stomach, and its expression has been associated with metastases and disease recurrence in gastric cancer [23]. We confirm this, for the first time, in adipocytes from CC tissues, where it is likely to play a similar role, being associated with tumor dissemination also in our study cohort. The similar, although not extremely high in quantitative terms, expression of generic markers of tissue inflammation and cell migration, like TNF $\alpha$ or MCP-1, suggests a superimposable presence of local inflamma- 
tion. The increased VEGF expression in EOC adipocytes supports the association of such pro-angiogenic factor with microvascular density in facilitating ovarian tumorigenesis and determining resistance to therapies [24]. This role of VEGF is reinforced by the fact that it is 10-20-fold more expressed in adipocytes of EOC than in controls.

The main novelty of our work is certainly the role played by the P2X7R-NLRP3 inflammasome platform, expressed similarly in CC and EOC, but strictly related to MCP-1 expression and peripheral WBC count. We have previously described a link between the inflammasome complex and MCP-1 expression in circulating lymphomonocytes of subjects with different levels of physical training [25]; the present results confer strength to the concept of the metabolic status of adipocytes as major determinant of macrophage inflammatory output in cancer. Production of MCP-1 from adipocytes promotes a direct recruitment of macrophages to adipose tissue; they may in turn secrete chemokines and cytokines, increasing local inflammatory and fibrotic responses and affecting adipocyte and neoplastic cells gene expression, including nuclear receptors like peroxisome proliferator-activated receptor gamma [26].

In our view, an observation deserving attention is the higher presence of the P2X7R-NLRP3 complex in patients carrying carcinosis. Previous reports have linked P2X7R, through MCP-1, to cytokine release and cell proliferation in experimental models of human glioma [27, 28]; this receptor was described already as more expressed in CC patients with poor prognosis [29]. Our observation is, to our knowledge, the first one linking such pattern to aggressive abdominal cancers, where the chemokine CCL2/MCP-1 may facilitate macrophage recruitment into the tumors [30]. In our study group, we were unable to point out a relationship between tumor grading and any of the measured inflammatory biomarker; we tend to attribute such negative result to the relatively small sample size and to the impossibility to directly compare grading between the 2 tumors; it would be interesting, however, to further test the existence of such association in larger casuistries.

We confirm here the role of TGF $\beta$ as marker of invasion and potential epithelial-mesenchymal transition in both CC, mainly via activating Smad pathway [31], and EOC, likely altering cell phenotype and increasing cell metabolism favorable for tumor invasion and metastasis [32]. In our study cohort, we do not observe a direct relation between adipocyte P2X7R and TGF $\beta$; however, a very recent intriguing report [33] has shown that $\mathrm{P} 2 \mathrm{X} 7 \mathrm{R}$ stimulation increases the TGF $\beta$-mediated epithelial-tomesenchymal transition in glioblastoma stem cells, suggesting a novel role for such still poorly explored path- way. Regarding FGFR1, a recognized marker of endometrial cancer [34], we describe here for the first time its increased expression in omental adipocytes of patients with metastatic CC and EOC, suggesting its clinical meaning in other female tumors. In this picture, it will be interesting to test the reproducibility of our observation also in CC developed in male individuals. In conclusion, these preliminary results suggest a putative role of the adipocyte P2X7R-NLRP3 inflammasome in modulating chemotaxis and metastatic spread in CC and EOC; further studies on larger groups of patients are required to better characterize the role of such pathway as prognosis determinant in solid neoplasms, as well as its similar or diverse role in the male sex.

\section{Statements of Ethics}

This prospective observational study was approved by the institutional review board of the University of Pisa (\#13831) and conducted ethically in accordance with the World Medical Association Declaration of Helsinki. All participants signed a written informed consent for the provision of biological material for research purposes.

\section{Conflict of Interest Statement}

The authors declare that they have no conflicts of interest to declare.

\section{Funding Sources}

This study has been supported by an institutional grant from the University of Pisa (PRA 2017).

\section{Author Contributions}

All authors contributed to the study conception and design. Material preparation, clinical and experimental data collection, and analysis were performed by A. Solini, L. Cobuccio, C. Rossi, F. Parolini, E. Biancalana, S. Cosio, M. Chiarugi, and A. Gadducci. The first draft of the manuscript was written by A. Solini, and all authors commented on previous versions of the manuscript. All authors read and approved the final manuscript.

\section{Data Availability Statement}

Data are available from the authors upon reasonable request. 


\section{References}

1 Bray F, Ferlay J, Soerjomataram I, Siegel RL, Torre LA, Jemal A. Global cancer statistics 2018: GLOBOCAN estimates of incidence and mortality worldwide for 36 cancers in 185 countries. CA Cancer J Clin. 2018;68(6):394424.

2 Nissan A, Stojadinovic A, Garofalo A, Esquivel J, Piso P. Evidence-based medicine in the treatment of peritoneal carcinomatosis: past, present and future. J Surg Oncol. 2009;100(4): $335-44$.

3 Lemoine L, Sugarbaker P, Van der Speeten K. Pathophysiology of colorectal peritoneal carcinomatosis: role of the peritoneum. World J Gastroenterol. 2016;22(34):7692-707.

4 Jayne D. Molecular biology of peritoneal carcinomatosis. Cancer Treat Res. 2007;134:2133.

5 Tan DS, Agarwal R, Kaye SB. Mechanisms of transcoelomic metastasis in ovarian cancer. Lancet Oncol. 2006;7(11):925-34.

6 Dolan RD, McSorley ST, Park JH, Watt DG, Roxburgh CS, Horgan PG, et al. The prognostic value of systemic inflammation in patients undergoing surgery for colon cancer: comparison of composite ratios and cumulative scores. Br J Cancer. 2018;119(1):40-51.

7 van Grevenstein WM, Hofland LJ, van Rossen ME, van Koetsveld PM, Jeekel J, van Eijck CH. Inflammatory cytokines stimulate the adhesion of colon carcinoma cells to mesothelial monolayers. Dig Dis Sci. 2007;52(10):277583.

8 Nagata $\mathrm{H}$, Ishihara S, Kishikawa J, Sonoda H, Murono K, Emoto S, et al. CD133 expression predicts post-operative recurrence in patients with colon cancer with peritoneal metastasis. Int J Oncol. 2018;52(3):721-32.

9 Oku T, Shimada K, Kenmotsu H, Ando Y, Kurisaka C, Sano R, et al. Stimulation of peritoneal mesothelial cells to secrete Matrix Metalloproteinase-9 (MMP-9) by TNF- $\alpha$ : a role in the invasion of gastric carcinoma cells. Int J Mol Sci. 2018;19(12):3961.

10 Adinolfi E, Giuliani AL, De Marchi E, Pegoraro A, Orioli E, Di Virgilio F. The P2X7 receptor: a main player in inflammation. Biochem Pharmacol. 2018;151:234-44.

11 Burnstock G, Knight GE. The potential of $\mathrm{P} 2 \mathrm{X} 7$ receptors as a therapeutic target, including inflammation and tumour progression. Purinergic Signal. 2018;14(1):1-18.

12 Zhang WJ, Hu CG, Zhu ZM, Luo HL. Effect of P2X7 receptor on tumorigenesis and its pharmacological properties. Biomed Pharmacother. 2020;125:109844.
13 Zhang Y, Ding J, Wang L. The role of P2X7 receptor in prognosis and metastasis of colorectal cancer. Adv Med Sci. 2019;64(2): 388-94.

14 Adinolfi E, Capece M, Franceschini A, Falzoni S, Giuliani AL, Rotondo A, et al. Accelerated tumor progression in mice lacking the ATP receptor P2X7. Cancer Res. 2015;75(4): 635-44.

15 Mikula-Pietrasik J, Uruski P, Szubert S, Maksin K, Moszynski R, Szpurek D, et al. The proangiogenic capabilities of malignant ascites generated by aggressive ovarian tumors. Biomed Res Int. 2017;2017:2592496.

16 Kim S, Gwak H, Kim HS, Kim B, Dhanasekaran DN, Song YS. Malignant ascites enhances migratory and invasive properties of ovarian cancer cells with membrane bound IL-6R in vitro. Oncotarget. 2016;7(50):83148-59.

17 Vázquez-Cuevas FG, Cruz-Rico A, Garay E, García-Carrancá A, Pérez-Montiel D, Juárez B, et al. Differential expression of the P2X7 receptor in ovarian surface epithelium during the oestrous cycle in the mouse. Reprod Fertil Dev. 2013;25(7):971-84.

18 American Joint Committee on Cancer. AJCC cancer staging manual. In: Edge SB, Byrd SR, Compton CC, April GF, Frederick LG, Andrew T, editors. 7th ed.New York, NY: Springer-Verlag; 2010. p. 143-64.

19 Prat J. Ovarian, fallopian tube and peritoneal cancer staging: rationale and explanation of new FIGO staging 2013. Best Pract Res Clin Obstet Gynaecol. 2015;29(6):858-69.

20 Rossi C, Santini E, Chiarugi M, Salvati A, Comassi M, Vitolo E, et al. The complex P2X7 receptor/inflammasome in perivascular fat tissue of heavy smokers. Eur J Clin Invest. 2014;44(3):295-302

21 Wei HJ, Zeng R, Lu JH, Lai WF, Chen WH, Liu HY, et al. Adipose-derived stem cells promote tumor initiation and accelerate tumor growth by interleukin- 6 production. Oncotarget. 2015;6(10):7713-26.

22 Yang C, Wei C, Wang S, Shi D, Zhang C, Lin $\mathrm{X}$, et al. Elevated CD163+/CD68+ ratio at tumor invasive front is closely associated with aggressive phenotype and poor prognosis in colorectal cancer. Int J Biol Sci. 2019;15(5): 984-98.

23 Inokuchi M, Murase H, Otsuki S, Kawano T, Kojima K. Different clinical significance of FGFR1-4 expression between diffuse-type and intestinal-type gastric cancer. World J Surg Oncol. 2017;15(1):2.
24 Avril S, Dincer Y, Malinowsky K, Wolff C, Gündisch S, Hapfelmeier A, et al. Increased PDGFR-beta and VEGFR-2 protein levels are associated with resistance to platinum-based chemotherapy and adverse outcome of ovarian cancer patients. Oncotarget. 2017;8(58): 97851-61.

25 Comassi M, Santini E, Rossi C, Vitolo E, Seghieri M, Tocchini L, et al. The level of physical training modulates cytokine levels through P2X7 receptor in healthy subjects. Eur J Clin Invest. 2018;48(2).

26 Sartipy P, Loskutoff DJ. Monocyte chemoattractant protein 1 in obesity and insulin resistance. Proc Natl Acad Sci U S A. 2003;100(12): 7265-70.

27 Fang KM, Wang YL, Huang MC, Sun SH, Cheng H, Tzeng SF. Expression of macrophage inflammatory protein-1 $\alpha$ and monocyte chemoattractant protein-1 in glioma-infiltrating microglia: involvement of ATP and P2X7 receptor. J Neurosci Res. 2011;89(2): 199-211.

28 Braganhol E, Kukulski F, Lévesque SA, Fausther M, Lavoie EG, Zanotto-Filho A, et al. Nucleotide receptors control IL-8/CXCL8 and MCP-1/CCL2 secretions as well as proliferation in human glioma cells. Biochim Biophys Acta. 2015;1852(1):120-30.

29 Qian F, Xiao J, Hu B, Sun N, Yin W, Zhu J. High expression of P2X7R is an independent postoperative indicator of poor prognosis in colorectal cancer. Hum Pathol. 2017;64:61-8.

30 Qian BZ, Li J, Zhang H, Kitamura T, Zhang J, Campion LR, et al. CCL2 recruits inflammatory monocytes to facilitate breast-tumour metastasis. Nature. 2011;475(7355):222-5.

31 Fuxe J, Vincent T, Garcia de Herreros A Transcriptional crosstalk between TGF- $\beta$ and stem cell pathways in tumor cell invasion: role of EMT promoting Smad complexes. Cell $\mathrm{Cy}$ cle. 2010;9(12):2363-74

32 Al Ameri W, Ahmed I, Al-Dasim FM, Ali Mohamoud Y, Al-Azwani IK, Malek JA, et al. Cell type-specific TGF- $\beta$ mediated EMT in $3 \mathrm{D}$ and $2 \mathrm{D}$ models and its reversal by TGF- $\beta$ receptor kinase inhibitor in ovarian cancer cell lines. Int J Mol Sci. 2019;20(14):3568.

33 Ziberi S, Zuccarini M, Carluccio M, Giuliani P, Ricci-Vitiani L, Pallini R, et al. Upregulation of epithelial-to-mesenchymal transition markers and $\mathrm{P} 2 \mathrm{X} 7$ receptors is associated to increased invasiveness caused by P2X7 receptor stimulation in human glioblastoma stem cells. Cells. 2019;9(1):85.

34 Ma X, Lakshmipriya T, Gopinath SCB. Recent advances in identifying biomarkers and highaffinity aptamers for gynecologic cancers diagnosis and therapy. J Anal Methods Chem. 2019;2019:5426974.
Solini/Cobuccio/Rossi/Parolini/ Biancalana/Cosio/Chiarugi/Gadducci 